The following pages constitute the final, accepted and revised manuscript of the article:

Luo G, Hurtig M, Zhang X, Nilsson-Ehle P, Xu N.

"Leptin inhibits apolipoprotein $\mathrm{M}$ transcription and secretion in human hepatoma cell line, HepG2 cells."

Biochim Biophys Acta. 2005 May 15;1734(2):198-202.

Publisher: Elsevier.

Use of alternative location to go to the published version of the article requires journal subscription.

Alternative location:

http://dx.doi.org/10.1016/j.bbalip.2005.02.005 
Elsevier Editorial System(tm) for BBA - Molecular and Cell Biology of Lipids

Manuscript Draft

Manuscript Number:

Title: Leptin Inhibits Apolipoprotein M Transcription and Secretion

In Human Hepatoma Cell Line, HepG2 Cells

Article Type: Regular Paper

Section/Category:

Keywords: Lipoprotein; Apolipoprotein M; Leptin; HepG2 cell line

Corresponding Author: Dr. Ning Xu,

Corresponding Author's Institution: University Hospital of Lund

First Author: Guanghua Luo, MSc

Order of Authors: Guanghua Luo, MSc; Maria Hurtig, B.S.; Xiaoying Zhang, MD;

Peter Nilsson-Ehle, MD, PhD; Ning Xu

Manuscript Region of Origin:

Abstract : ABSTRACT

Apolipoprotein M (apoM) is a novel apolipoprotein presented mostly in highdensity lipoprotein (HDL) in human plasma. Previously we have reported that both leptin and leptin receptor are essential for apoM expression in vivo. Expression of apoM is lower in the leptin deficient (ob/ob) mouse and leptin receptor deficient $(\mathrm{db} / \mathrm{db})$ mouse than in the normal mouse. In the present study, however, we demonstrated that supra-physiological concentrations of recombinant leptin significantly inhibited apoM transcription and secretion in the human hepatoma cell line, HepG2 cells. Both northern blotting and real-time RT-PCR were applied into the analyses of apoM mRNA levels, and compatible data were obtained. The inhibitory effect of leptin on apoM mRNA levels in HepG2 cells is dose dependent, i.e. $100 \mathrm{ng} / \mathrm{mL}$ of leptin decreased apoM mRNA levels by 30\%, and 500 $\mathrm{ng} / \mathrm{mL}$ of leptin decreased apoM mRNA levels about 50\%. Even at a physiological concentration of leptin $(10 \mathrm{ng} / \mathrm{mL})$, apoM expression was decreased, and in parallel, secretion of apoM into the medium was also decreased. Furthermore, we examined apoAI, apoB and apoE by northern blotting analyses. The results demonstrated that leptin does not significantly influence expressions of apoAI, apoB and apoE in Hepc2 cells, suggesting that leptin has a specific regulatory effect on hepatic apoM transcription and secretion in vitro. The mechanism on the contradictory effects of leptin on apom expression in vivo and in vitro needs further investigation. 
January 20, 2005

Prof. F. Spener

Executive Editor, BBA-Molecular and Cell Biology of Lipids

Dear Prof. Spener,

Enclosed please find the manuscript "Leptin Inhibits Apolipoprotein M Transcription and Secretion In Human Hepatoma Cell Line, HepG2 Cells" by Guanghua Luo, Maria Hurtig, Xiao-Ying Zhang, Peter Nilsson-Ehle and myself to be considered for publication in the BBA-Molecular and Cell Biology of Lipids as a regular paper. All authors have read and approved the manuscript. There has been no duplicate publication or submission elsewhere of any part of the work. There are no financial or other relations that could lead to a conflict of interest.

Sincerely yours,

Ning $\mathrm{Xu}, \mathrm{MD}, \mathrm{PhD}$.

Department of Clinical chemistry

Institute of Laboratory Medicine

University Hospital of Lund

S-221 85 Lund

Sweden

Tel: $+46-46173462$

Fax. $+46-46130064$

Email: ning.xu@klinkem.lu.se 


\title{
Leptin Inhibits Apolipoprotein M Transcription and Secretion In Human Hepatoma Cell Line, HepG2 Cells
}

Guanghua Luo§ף, Maria Hurtigף, Xiaoying Zhang§, Peter Nilsson-Ehleף, and Ning Xuף*

\Department of Clinical Chemistry, Institute of Laboratory Medicine, University

Hospital of Lund, S-221 85 Lund, Sweden, and §Laboratory of Molecular Medicine, The Third Affiliated Hospital, Suzhou University, Changzhou 213003, China

Short title: Leptin decreases apoM synthesis in HepG2 cells

\author{
*Corresponding person: \\ Ning Xu, MD, PhD \\ Department of Clinical Chemistry \\ Institute of Laboratory Medicine \\ University Hospital of Lund \\ S-221 85 Lund, Sweden
}

Tel: $+46-46173462$

Fax: +46-46130064

Email: ning.xu@klinkem.lu.se 


\begin{abstract}
Apolipoprotein M (apoM) is a novel apolipoprotein presented mostly in high-density lipoprotein (HDL) in human plasma. Previously we have reported that both leptin and leptin receptor are essential for apoM expression in vivo. Expression of apoM is lower in the leptin deficient $(o b / o b)$ mouse and leptin receptor deficient $(d b / d b)$ mouse than in the normal mouse. In the present study, however, we demonstrated that supra-physiological concentrations of recombinant leptin significantly inhibited apoM transcription and secretion in the human hepatoma cell line, HepG2 cells. Both northern blotting and realtime RT-PCR were applied into the analyses of apoM mRNA levels, and compatible data were obtained. The inhibitory effect of leptin on apoM mRNA levels in HepG2 cells is dose dependent, i.e. $100 \mathrm{ng} / \mathrm{mL}$ of leptin decreased apoM mRNA levels by $30 \%$, and 500 $\mathrm{ng} / \mathrm{mL}$ of leptin decreased apoM mRNA levels about $50 \%$. Even at a physiological concentration of leptin (10 ng/mL), apoM expression was decreased, and in parallel, secretion of apoM into the medium was also decreased. Furthermore, we examined apoAI, apoB and apoE by northern blotting analyses. The results demonstrated that leptin does not significantly influence expressions of apoAI, apoB and apoE in HepC2 cells, suggesting that leptin has a specific regulatory effect on hepatic apoM transcription and secretion in vitro. The mechanism on the contradictory effects of leptin on apoM expression in vivo and in vitro needs further investigation.
\end{abstract}

Key words: $\quad$ Lipoprotein; Apolipoprotein M; Leptin; and HepG2 cell line. 


\section{INTRODUCTION}

Apolipoprotein $\mathrm{M}(\mathrm{apoM})$ is a recently discovered human apolipoprotein that is associated mainly with high-density lipoprotein (HDL) in human plasma, it is also present in triglyceride-rich lipoproteins (TGRLP) and low-density lipoprotein (LDL) [1, 2]. In situ hybridization experiments demonstrated that apoM is exclusively expressed in hepatocytes in liver and in tubular cells in kidney [3, 4]. Previous studies suggest that apoM might be involved in lipid and/or lipoprotein metabolism in vivo. For example, the proportion of apoM in TGRLP has been shown to be increased in the postprandial phase [1]. Recently we demonstrated that both leptin and leptin receptor are essential for the apoM synthesis in vivo [5]. In the leptin deficient (ob/ob) mouse and leptin receptor deficient $(d b / d b)$ mouse the apoM mRNA levels and plasma apoM concentrations were greatly reduced when compared to the normal mouse. Furthermore, it is also reported that plasma apoM concentration is positively correlated to leptin level in obese subjects [6]. Liang and Tall reported that leptin treatment in $o b / o b$ mice leads to an increase in mRNA levels of apoAI, apoAII, apoH and apoM, and a decrease in apoAIV in the liver [7]. In the present study, we investigated the effects of leptin on the transcription and secretion of apoM in HepG2 cells. We chose HepG2 cells as these cells have a known leptin receptor [8]. Also, the HepG2 cells can synthesize and secrete lipoprotein including very lower density lipoprotein (VLDL), intermediate density lipoprotein (IDL) and HDL, as

well as a variety of plasma apolipoproteins including apoB, apoE, apoAs, apoCs and apoM. 


\section{MATERIALS AND METHODS}

\section{Materials.}

Recombinant leptin was purchased from SIGMA Chemical Company (St. Louis, MO, USA) and from Research Diagnostics, Inc (Flanders, NJ, USA). Oleic acid was from Larodan Fine Chemicals, Sweden. The established hepatoblastoma cell line, HepG2, was from American Type Culture Collection, Manassas, VA. $\left[{ }^{32} \mathrm{P}\right] \mathrm{dCTP}$ was purchased from Amersham Pharmacia Biotech. Six-well cell culture clusters and $25-\mathrm{cm}^{2}$ vented cell culture flasks were purchased from Costar. Real-time RT-PCR reagents and control probe G3PDH were purchased from PE Biosystems.

\section{Cell cultures.}

HepG2 cells were maintained in RPMI 1640 with $10 \%$ fetal calf serum (FCS) in the presence of benzylpenicillin $(100 \mathrm{U} / \mathrm{mL})$ and streptomycin $(100 \mu \mathrm{g} / \mathrm{mL})$ under standard culture conditions $\left(5 \% \mathrm{CO}_{2}, 37^{\circ} \mathrm{C}\right)$. Cells were seeded in $25-\mathrm{cm}^{2}$ cell culture flasks or in 6-well cell culture clusters, and allowed growing to 50-70\% confluence. Prior to the experiment, cells were washed twice with phosphate buffered saline (PBS), once with serum free RPMI 1640, and then the experimental medium (RPMI 1640+0.5\% human serum albumin (HSA) + antibiotics) containing different leptin concentrations. Cells were incubated at $37^{\circ} \mathrm{C}$ for $24 \mathrm{hrs}$.

Preparation of northern blotting probes.

A commercial G3PDH cDNA fragment was used as a control probe and a full-length human apoM cDNA was used as a probe for apoM mRNA hybridization [1]. A 651 bp apoAI exon 3 DNA fragment was amplified from human genomic DNA (primers: 5'ctgggacagcgtgacctc and 5'-tctgagcaccgggaaggg) as a probe for apoAI hybridization, and 
a 317 bp DNA fragment of the apoE gene was amplified from human genomic DNA (primers: 5'-ctgatggacgagaccatgaa and 5'-tcgcgggecccggectggta) for apoE hybridization. A 385 bp DNA fragment corresponding to the apoB LDL-R binding domain was amplified (primers: 5'-gctgtactctaccgctaaagga and 5'-gacatttgccatggagagagtt) from human genomic DNA, and used as the human apoB northern blotting probe [9]. All PCR reactions were performed using Ampli Taq DNA polymerase with buffers and dNTPs supplied by manufacturer (Perkin Elmer, Roche Molecular Systems Inc., USA) according to the manufacture's directions, on a GeneAmp PCR System 2400 (Perkin Elmer, Applied Biosystems). All probes were radiolabeled with $\left[{ }^{32} \mathrm{P}\right] \mathrm{dCTP}$ using the random primer method (RediPrime, Amersham Pharmacia Biotech). Isolation of total RNA from HepG2 cells and northern blotting analysis. Total RNA of HepG2 cells was isolated by the guanidinium thiocyanate method [10] and northern blots were hybridized with the probes described above and eventually exposed to X-ray film. Hybridizations were carried out at $65^{\circ} \mathrm{C}$ in a hybridization solution (Clontech). The blots were washed several times in $2 \mathrm{X} \mathrm{SSC} \mathrm{/} \mathrm{0.1 \%} \mathrm{SDS} \mathrm{solution} \mathrm{at} \mathrm{room}$ temperature for $2 \mathrm{hrs}$ and twice in $0.1 \mathrm{X} \mathrm{SSC} / 0.05 \% \mathrm{SDS}$ at $50{ }^{\circ} \mathrm{C}$ for $40 \mathrm{~min}$. The washed blots were exposed to X-ray film at $-70{ }^{\circ} \mathrm{C}$ from 1-3 days. The autoradiographs were analyzed with a scanner (Epson-1600). The membrane was stripped with boiled water in the presence of $0.5 \%$ SDS for 10 min and then rehybridized with the next probe. The relative mRNA levels were calculated with a Macintosh computer using the software of Quantity One (Version 4.2.1, Bio-Rad Laboratories) and presented as volume (intensity $\mathrm{x} \mathrm{mm}^{2}$ ). 


\section{Real time RT-PCR.}

Primer Express software (Applied Biosystems) was used to design human apoM primers and probe used in the TaqMan assay. In order to avoid amplify DNA template the apoM specific primers that are spanned an $81 \mathrm{bp}$-intron were designed to amplify a 66-bp product. The primers were 5'-tgccccggaaatggatcta and 5'-cagggcggccttcagtt, and the probe was 5'-FAM-cacctgactgaagggagcacagatctca-TAMRA. A G3PDH primer/probe set (PE Biosystems) was used in separate tubes as control for the input cDNA. Relative standard curve was performed to compensate the efficiency of the PCR. In brief, a serial dilution of human apoM cDNA was used to generate a standard curve by plotting the cycle threshold versus the log of input cDNA. Both G3PDH standard curve and apoM standard curve were linear. Relative quantification of mRNA was performed on an ABI PRISM 7700 Sequence Detector (Applied Biosystems), as described previously [11]. Real time RT-PCR of apoM was performed in two-steps. It was carried out in a $25 \mu 1$ reaction mixture containing 1x TaqMan Universal PCR Master Mix, 22.5 pmol of both forward and reverse primers, 5 pmol probe and $50 \mathrm{ng}$ of the total RNA templates. Thermal cycling conditions included the following steps: $25^{\circ} \mathrm{C} 10 \mathrm{~min}, 48^{\circ} \mathrm{C} 30 \mathrm{~min}$ and $95{ }^{\circ} \mathrm{C} 5 \mathrm{~min}$ to do reverse transcription, and then the reaction mixture was preheated for $2 \mathrm{~min}$ at $50{ }^{\circ} \mathrm{C}$ and for $10 \mathrm{~min}$ at $95{ }^{\circ} \mathrm{C}$ to activate Taq polymerase. After that, a 40 cycle two-step PCR was performed consisting of $15 \mathrm{sec}$ at $95^{\circ} \mathrm{C}$ and $1 \mathrm{~min}$ at $60^{\circ} \mathrm{C}$. Samples were amplified simultaneously in triplicates in one-assay run. Real-time RTPCR of G3PDH was performed according to the manufacturer's instruction. The threshold cycle $\left(\mathrm{C}_{\mathrm{T}}\right)$ is defined as the fractional cycle number at which the reporter fluorescence reaches a certain level. 
ApoAI, apoB, apoE and apoM protein masses determinations.

ApoAI, apoB, apoE and apoM levels were determined by western blotting analysis [9]. Rabbit antiserum against a synthetic peptide corresponding to amino acid residues 103122 of apoM, or truncated apoM conjugated to keyhole limpet hemocyanin (LKH) was raised. Cell culture medium without FCS was fractionated by SDS-polyacrylamide gel electrophoresis (SDS-PAGE), transferred to nitrocellulose membrane, and then incubated with polyclonal rabbit against human apoAI, apoB, apoE or apoM antibodies. Alkaline phosphatase-conjugated swine anti rabbit IgG was used as the secondary antibody. Bands corresponding to the individual apolipoproteins were visualized by an AP Conjugate Substrate Kit (Bio-Rad Laboratories), and quantified by a scanner using the software of Quantity One.

Statistics.

Results are expressed as mean $\pm \mathrm{SD}$. Comparisons among groups were statistically evaluated by the one-way ANOVA and followed by the unpaired student $t$-test. Significance was established at a $\mathrm{p}$ value less than 0.05 . 


\section{RESULTS}

Effect of leptin on apoAI, apoB, apoE and apoM secretion

The contents of apoM in the medium secreted from HepG2 cells exposed to leptin for 24 hrs at different concentrations are shown in Fig. 1. At leptin concentrations of 100 and $500 \mathrm{ng} / \mathrm{ml}$, apoM levels decreased by $20 \%$ and $23 \%$, respectively. There were no significant changes in the secretion of apoAI, apoB and apoE into the medium after any leptin administration in HepG2 cell cultures (data not shown).

Effects of leptin on apoAI, apoB, apoE and apoM expression detected by northern blotting analysis

Fig 2 demonstrates that leptin significantly decreased apoM mRNA levels in HepG2 cells. The effects were dose dependent. At the highest dosage of leptin $(500 \mathrm{ng} / \mathrm{ml})$, the reduction of apoM mRNA levels was $58 \%$ compared to the control cells, but already at physiological concentration of leptin $(10 \mathrm{ng} / \mathrm{mL})$ apoM expression was slightly inhibited. There were no obvious changes in mRNA levels of apoAI, apoB, apoE or control probe, G3PDH, during the administration of leptin (Fig 2).

\section{Measurement of apoM mRNA levels by Real-time RT-PCT}

Fig 3 demonstrated that the relative apoM mRNA levels were significantly decreased after administrations of leptin at both 100 and $500 \mathrm{ng} / \mathrm{mL}$, as well as at physiological concentrations of leptin $(10 \mathrm{ng} / \mathrm{ml})$. The effects of leptin on apoM expression in HepG2 cells were dose-dependent. The Fig. 3 represents one of four similar experiments. 


\section{DISCUSSION}

ApoM is a newly discovered human HDL associated apolipoprotein. The pathophysiological function of apoM is not yet understood. Recently we demonstrated that both leptin and leptin receptor are essential for the apoM expression in vivo [5]. Expression of apoM was lower in the leptin deficient $(o b / o b)$ mouse and leptin receptor deficient $(d b / d b)$ mouse than in the normal mouse. We have also reported that apoM concentration in plasma was positively correlated to leptin level in obese subjects [6]. In the present study, we further examined the effects of leptin on apoM synthesis in HepG2 cell cultures. It is demonstrated that leptin inhibited apoM expression and secretion in the HepG2 cells. Two different methods were applied to measure the relative apoM expressions, i.e., northern blotting and real-time RT-PCR. Both analyses display similar data demonstrating that supra-physiological concentrations of leptin (ten to fifty-times physiological concentration) significantly inhibited apoM transcription in HepG2 cell cultures. The inhibitory effect of leptin on apoM mRNA transcription and secretion in HepG2 cells was dose dependent, whereas leptin did obviously influence neither secretion nor expression of apoAI, apoB and apoE in $\mathrm{HepC} 2$ cells. This indicates that leptin has a specific regulatory effect on hepatic apoM transcription and secretion in vitro.

Leptin is a cytokine-like peptide secreted by adipose tissue, known to regulate hepatic lipid and lipoprotein metabolism [12-15]. Mice with genetic defects in leptin exhibit hyperlipidemia, hyperinsulinemia, hyperglycemia and resistance to insulin [16-18]. Administration of leptin could partially or fully reverse these abnormal phenotypes [12, 
$13,15,19-21]$. It is still unknown why leptin has contradictory effects on apoM expression in vivo and in vitro. One possible explanation is that leptin is a multifunctional hormone that regulates many metabolic aspects in vivo.

ApoM is mainly associated with HDL with a small proportion present in TGRLP and LDL in human plasma [1]. On a human tissue-array blotting analysis, apoM mRNA was expressed exclusively in liver and in kidney, with a minor expression found in fetal liver and in fetal kidney [22]. The synthesis of apoM in liver may be associated with the production of HDL, whereas the physiological importance of the apoM mRNA in kidney is less obvious. Apart from the hypothetical involvement of apoM in the synthesis of HDL, it is possible that apoM serves a specific function in the metabolism and transfer of cholesterol and/or other bioactive molecules in plasma. Molecular modeling methods demonstrated that apoM is a member of the lipocalin super-family [23]. Most members of this super-family have the ability to bind and transport hydrophobic molecules in plasma. Alternatively, apoM could have the ability to transport hydrophobic low molecular weight compounds, the nature of which remains to be determined. 


\section{Acknowledgements:}

Anette Jutzeler, Gerd Nilsson and Siv Svensson provided excellent technical assistance.

This work was supported by grants from the Physiographic Society of Lund, the Medical

Faculty, University of Lund, Påhlsson’s Foundation, OE \& Edla Johansson’s Scientific

Foundation and the Swedish Medical Research Council (project no. 04966). 


\section{LEGEND TO FIGURES}

\section{Fig 1.}

Effect of leptin on apoM secretion in HepG2 cells. ApoM concentration was determined by western blotting analysis as described in the material and methods. Data are expressed as the volume that was analyzed by the software of Quantity One. Data are means \pm SD ( $\mathrm{n}=6$ for each sample group). Lanes $1-3$, control group, lanes 4-12, leptin concentrations at 10,100 and $500 \mathrm{ng} / \mathrm{mL}$, respectively. ${ }^{*} \mathrm{P}<0.05$ vs. control group.

\section{Fig 2.}

Northern blotting analysis of G3PDH, apoAI, apoB, apoE and apoM mRNA levels after administration of leptin. Total RNA was extracted, and mRNA levels of G3PDH, apoAI, apoB, apoE and apoM were determined. Aliquots of $10 \mu \mathrm{g}$ total RNA isolated from control cells (without leptin) or cells treated with different amounts of leptin (10$500 \mathrm{ng} / \mathrm{mL}$ ), were used. The same membrane was blotted with ${ }^{32} \mathrm{P}$-labeled probes. Data are means $\pm \mathrm{SD}$ ( $\mathrm{n}=5$ for each sample group). $* * \mathrm{P}<0.01$ vs. control group.

\section{Fig 3.}

Real-time RT-PCR determination of apoM levels in control cells and in leptin treated cells. ApoM mRNA levels were determined by real-time RT-PCR analyses as described in the materials and methods. Each experimental group contains 6 replicates and realtime RT-PCT was run triplicates. Data are means $\pm \mathrm{SD}(\mathrm{n}=6$ for each sample group). Control group represented $100 \%$. $* \mathrm{P}<0.05 ; * * \mathrm{P}<0.01$ vs. control group. The data is one of four similar experiments. 


\section{REFERENCES:}

[1] N. Xu and B. Dahlback, A novel human apolipoprotein (apoM), J Biol Chem 274 (1999) 31286-90.

[2] G. Luo, X. Zhang, P. Nilsson-Ehle and N. Xu, Apolipoprotein M, Lipids Health Dis 3 (2004) 21.

[3] X.Y. Zhang, X. Dong, L. Zheng, G.H. Luo, Y.H. Liu, U. Ekström, P. NilssonEhle, Q. Ye and N. Xu, Specific tissue expression and cellular localization of human apolipoprotein $\mathrm{M}$ as determined by in situ hybridization., Acta Histochemica (2002).

[4] X.Y. Zhang, G.Q. Jiao, M. Hurtig, X. Dong, L. Zheng, G.H. Luo, P. Nilsson-Ehle, Q. Ye and N. Xu, Expression pattern of apolipoprotein M during mouse and human embryogenesis, Acta Histochem 106 (2004) 123-8.

[5] N. Xu, P. Nilsson-Ehle, M. Hurtig and B. Ahren, Both leptin and leptin-receptor are essential for apolipoprotein M expression in vivo, Biochem Biophys Res Commun 321 (2004) 916-21.

[6] N. Xu, P. Nilsson-Ehle and B. Ahren, Correlation of apolipoprotein M with leptin and cholesterol in normal and obese subjects, J Nutr Biochem 15 (2004) 579-82.

[7] C.P. Liang and A.R. Tall, Transcriptional profiling reveals global defects in energy metabolism, lipoprotein, and bile acid synthesis and transport with reversal by leptin treatment in ob/ob mouse liver, J Biol Chem 276 (2001) 49066-76.

[8] S. Kaser, B. Foger, C.F. Ebenbichler, R. Kirchmair, R. Gander, A. Ritsch, A. Sandhofer and J.R. Patsch, Influence of leptin and insulin on lipid transfer 
proteins in human hepatoma cell line, HepG2, Int J Obes Relat Metab Disord 25 (2001) 1633-9.

[9] N. Xu, U. Ekstrom and P. Nilsson-Ehle, Acth decreases the expression and secretion of apolipoprotein b in hepg2 cell cultures, J Biol Chem 276 (2001) 38680-4.

[10] P. Chomczynski and N. Sacchi, Single-step method of RNA isolation by acid guanidinium thiocyanate- phenol-chloroform extraction, Anal Biochem 162 (1987) 156-9.

[11] N. Xu, M. Hurtig, X.Y. Zhang, Q. Ye and P. Nilsson-Ehle, Transforming growth factor-beta down-regulates apolipoprotein M in HepG2 cells, Biochim Biophys Acta 1683 (2004) 33-7.

[12] S. VanPatten, N. Ranginani, S. Shefer, L.B. Nguyen, L. Rossetti and D.E. Cohen, Impaired biliary lipid secretion in obese Zucker rats: leptin promotes hepatic cholesterol clearance, Am J Physiol Gastrointest Liver Physiol 281 (2001) G393404.

[13] N. Matsuoka, Y. Ogawa, H. Masuzaki, K. Ebihara, M. Aizawa-Abe, N. Satoh, E. Ishikawa, Y. Fujisawa, A. Kosaki, K. Yamada, H. Kuzuya and K. Nakao, Decreased triglyceride-rich lipoproteins in transgenic skinny mice overexpressing leptin, Am J Physiol Endocrinol Metab 280 (2001) E334-9.

[14] D.L. Silver, X.C. Jiang and A.R. Tall, Increased high density lipoprotein (HDL), defective hepatic catabolism of ApoA-I and ApoA-II, and decreased ApoA-I mRNA in ob/ob mice. Possible role of leptin in stimulation of HDL turnover, J Biol Chem 274 (1999) 4140-6. 
[15] J. Lopez-Soriano, N. Carbo, F.J. Lopez-Soriano and J.M. Argiles, Short-term effects of leptin on lipid metabolism in the rat, FEBS Lett 431 (1998) 371-4.

[16] D. Shmulewitz, S.B. Auerbach, T. Lehner, M.L. Blundell, J.D. Winick, L.D. Youngman, V. Skilling, S.C. Heath, J. Ott, M. Stoffel, J.L. Breslow and J.M. Friedman, Epidemiology and factor analysis of obesity, type II diabetes, hypertension, and dyslipidemia (syndrome X) on the Island of Kosrae, Federated States of Micronesia, Hum Hered 51 (2001) 8-19.

[17] C. Vigouroux, S. Gharakhanian, Y. Salhi, T.H. Nguyen, D. Chevenne, J. Capeau and W. Rozenbaum, Diabetes, insulin resistance and dyslipidaemia in lipodystrophic HIV-infected patients on highly active antiretroviral therapy (HAART), Diabetes Metab 25 (1999) 225-32.

[18] F.C. de la Brousse, B. Shan and J.L. Chen, Identification of the promoter of the mouse obese gene, Proc Natl Acad Sci U S A 93 (1996) 4096-101.

[19] J. Auwerx and B. Staels, Leptin, Lancet 351 (1998) 737-42.

[20] P.J. Havel, Control of energy homeostasis and insulin action by adipocyte hormones: leptin, acylation stimulating protein, and adiponectin, Curr Opin Lipidol 13 (2002) 51-9.

[21] K. Reue and M. Peterfy, Mouse models of lipodystrophy, Curr Atheroscler Rep 2 (2000) 390-6.

[22] X.Y. Zhang, X. Dong, L. Zheng, G.H. Luo, Y.H. Liu, U. Ekstrom, P. NilssonEhle, Q. Ye and N. Xu, Specific tissue expression and cellular localization of human apolipoprotein $\mathrm{M}$ as determined by in situ hybridization, Acta Histochem 105 (2003) 67-72. 
[23] J. Duan, B. Dahlback and B.O. Villoutreix, Proposed lipocalin fold for apolipoprotein $\mathrm{M}$ based on bioinformatics and site-directed mutagenesis, FEBS Lett 499 (2001) 127-32. 
Figure 1
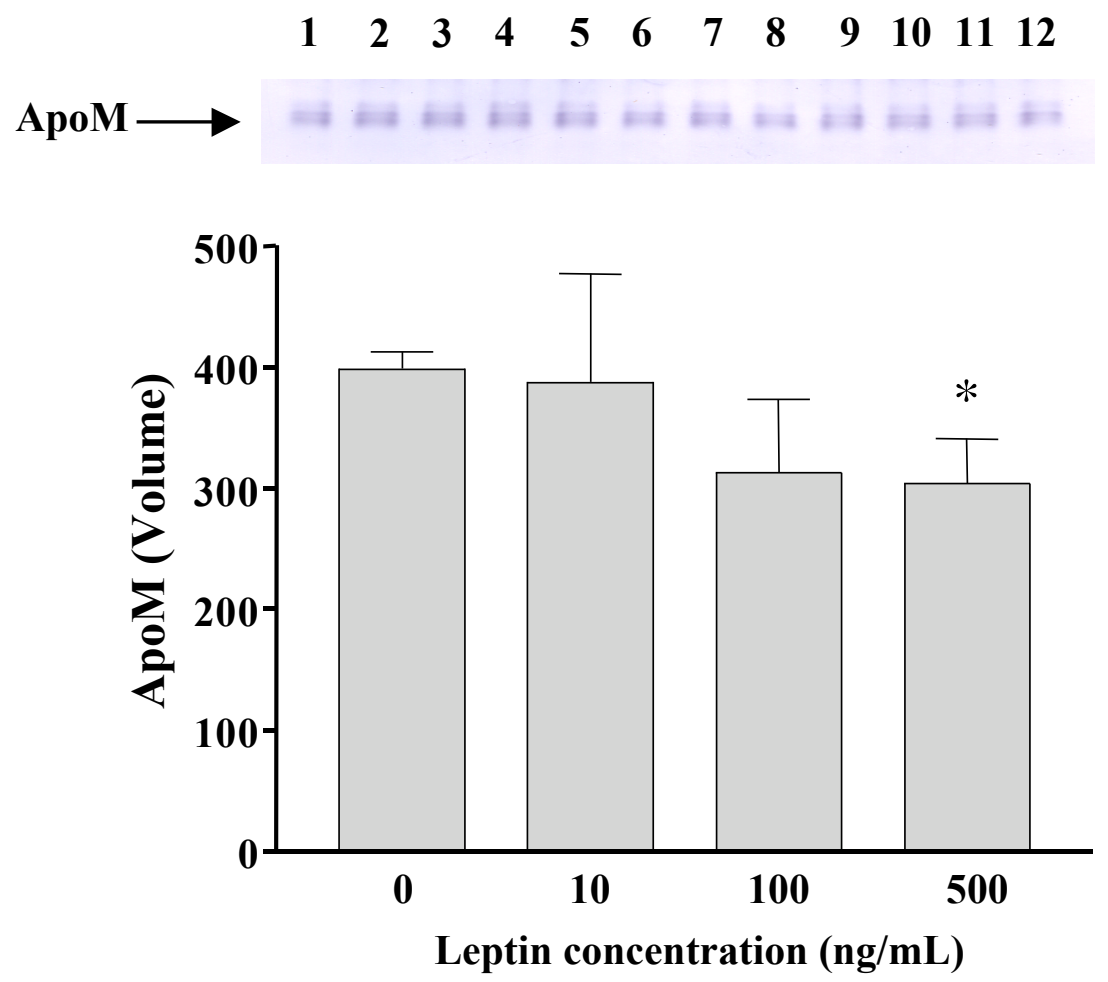
Figure 2

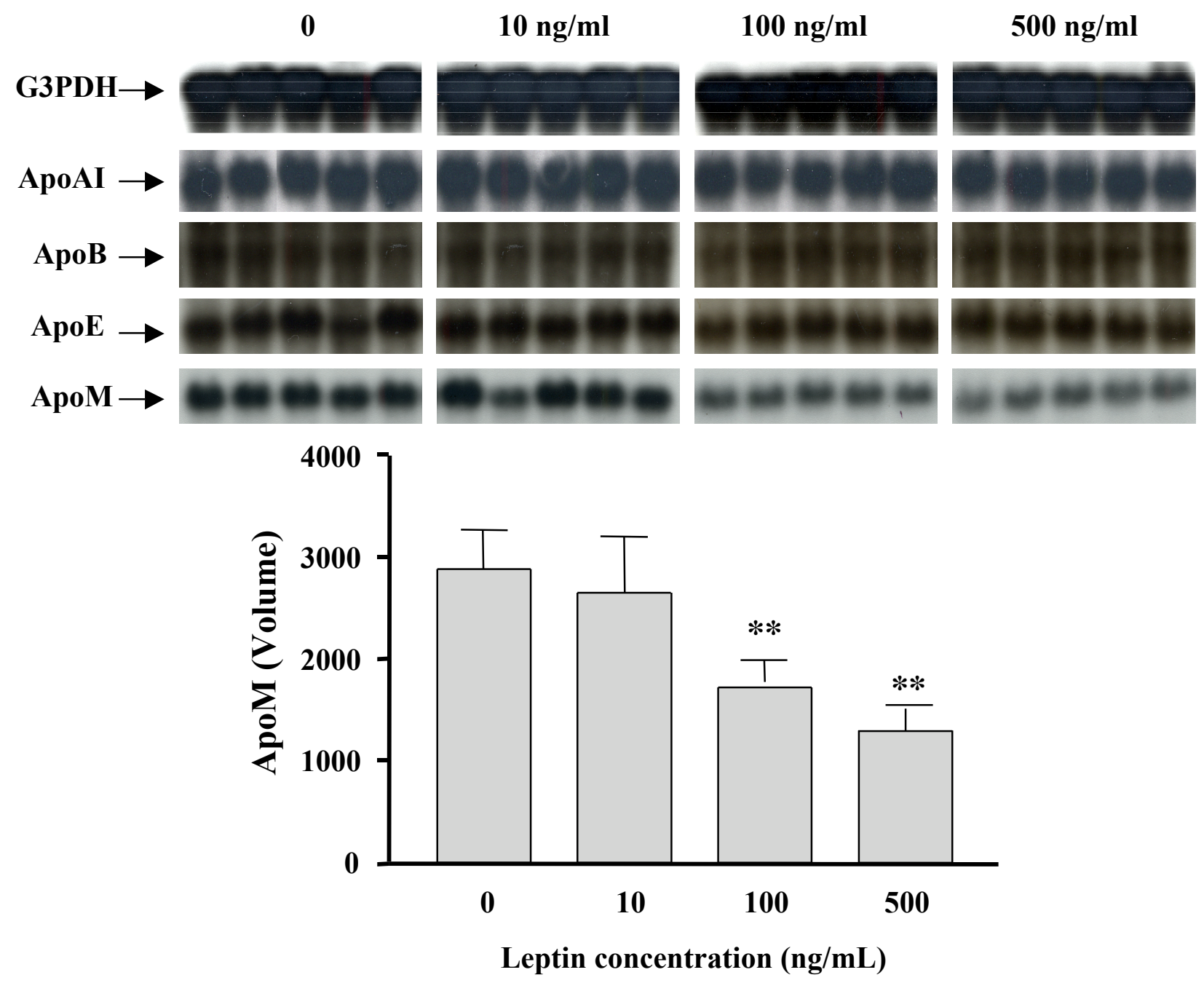


Figure 3

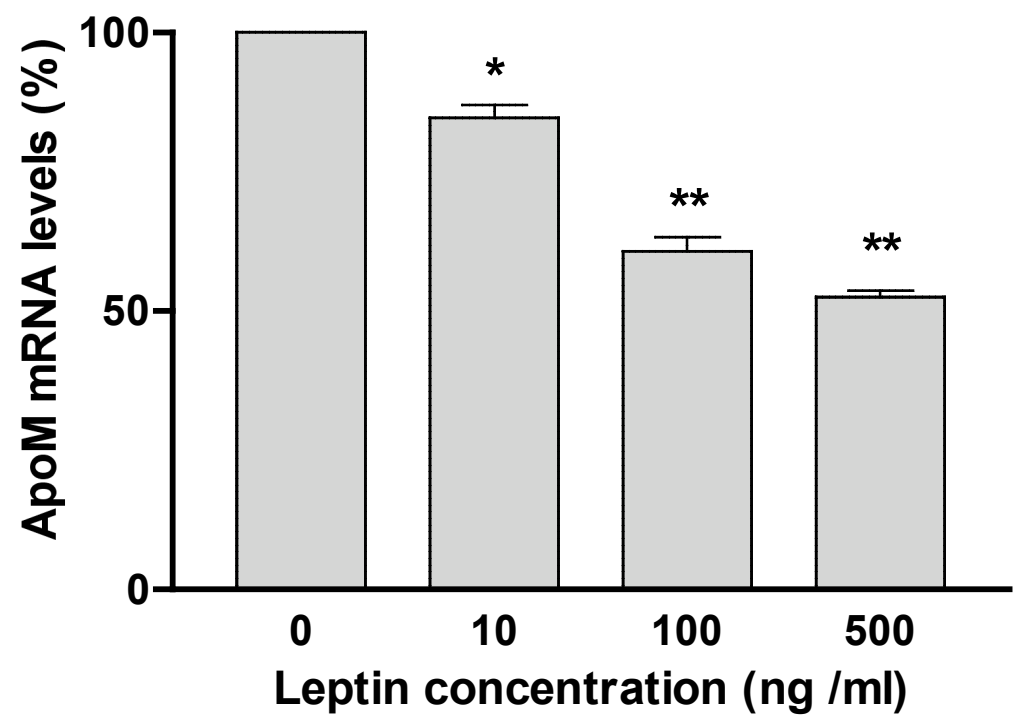




\section{Suggested reviewers:}

Olivecrona, Gunilla

Umea University

Bldg 6M, 3rd floor

Umea, SE-90187

Sweden

Gunilla.olivecrona@medbio.umu.se

Ole Faergeman

Aarhus Amtssygehus

University Hospital

DK-8000 Aarhus C

E-mail: ole.faergeman@aas.auh.dk

\section{Stoffell M}

Rockefeller Univ

Lab Metab Dis

1230 York Ave,Box 292

New York, NY 10021

USA

Camejo, German

AstraZeneca

Pepparedsleden 1

Molndal, S-431 83

Sweden

german.camejo@AstraZeneca.com 